Table 1. Sensitivities and specificities of examinations in gout and calcium pyrophosphate deposition disease

\begin{tabular}{lrrr}
\hline & DECT $\mathbf{n}=\mathbf{2 6}$ & US $\mathbf{n = 2 6}$ & $\begin{array}{c}\text { Conventional } \\
\text { Suspected clinical } \\
\text { Diagnosis } \mathbf{n = 2 6}\end{array}$ \\
\hline Gout & & & \\
Sensitivity & $66.67 \%(0.410-0.867)$ & $88.9 \%(0.652-0.986)$ & $61.5 \%(0.316-0.861)$ \\
Specificity & $87.5 \%(0.473-0.997)$ & $75 \%(0.349-0.968)$ & $100 \%(0.541-1.000)$ \\
CPPD & $62.5 \%(0.245-0.915)$ & $87.5 \%(0.523-0.936)$ \\
Sensitivity & $83.3 \%(0.586-0.964)$ & $88.9 \%(0.653-0.986)$ & $0 \%(0.000-0.459)$ \\
Specificity & & $62.5 \%(0.245-0.915)$ \\
\end{tabular}

${ }^{*} 95 \% \mathrm{Cl}$ in brackets

written content designed to fill information gaps around AAV diagnosis, investigations and treatment and what to expect during clinical follow up. The platform supports patients in asking questions and seeking information while signposting them to their own healthcare professional for advice and their local country patient association for support.

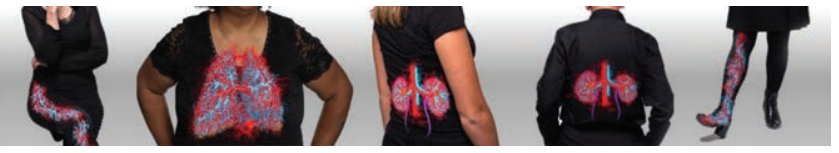

Conclusion: People with AAV need support throughout life, the profound psychosocial influence from illness makes the lived experience, challenging. SEE ME. HEAR ME online patient platform aims to generate awareness around AAV, improve physician and patient dialog, and enhance people's experiences of living and coping with the disease. In addition it provides support for carers and giving valuable insights to friends, family and the general public about what the lived experience with AAV looks like.

Acknowledgments: We wish to thank all European patients and patient association leads who worked on this project

Disclosure of Interests: Shanali Perera Consultant of: Vifor Pharma, Dijana krafcsik Employee of: Vifor Pharma, Peter Rutherford Shareholder of: Vifor Pharma, Employee of: Vifor Pharma, Baxter Healthcare DOI: 10.1136/annrheumdis-2020-eular.1494

\section{OP0320-PARE FIBROMYALGIA NETWORK - A MULTIDIMENSIONAL PROJECT FOR PEOPLE WITH FIBROMYALGIA SYNDROME}

A. Celano ${ }^{1}$, S. Mingolla ${ }^{2}$, I. Cinieri ${ }^{3}$, A. Marsico ${ }^{4} .{ }^{1} A P M A R R$, Lecce, Italy; ${ }^{1}$ APMARR, Lecce, Italy; ${ }^{3}$ Urbino University, Urbino, Italy; ${ }^{4}$ TA LHA, Taranto, Italy

Background: Fibromyalgia Syndrome (FMS) is a clinical non-joint syndrome characterized by diffuse, prolonged, and unexplained muscle pains. The health effects of FMS are pervasive and wide ranging. It is frequently associated with depression, anxiety and post-traumatic stress disorder. Patients describe living with daily unrelenting chronic widespread pain, persistent chronic fatigue, sleep issues, and cognitive effects. Fibromyalgia impacts all aspects of patients' lives. Patients report severe limitations in maintaining relationships, performing at work or school, and caring for self and family. They experience stigma within society, social isolation, healthcare and financial challenges of treatment, and fears of living with an often misunderstood condition. In 2019 APMARR launched Fibromyalgia Network a project that aimed at improving the quality of life of patients with FMS.

Objectives:

- Experiment a set of good practices at territorial level to be expanded nationally to improve the FMS patients' conditions

- Create a multisectoral, patient-oriented network with different stakeholders

- Launch a communication campaign to inform and change the perception of the pathology

- Train the General Practitioners in order to increase early diagnosis

- Empower the Patients

- Promote innovative treatments and the data acquisition about hyperbaric oxygen therapy

- Provide free psychological and information support

Methods: The project was based on the assumption supported by evidence that a multi-modal treatment approach improves the quality of life of person with FMS including a combination of drug and non-drug treatments and a range of health care specialties. All the activities implemented were shaped on a holistic approach to treating Fibromyalgia, including lifestyle management, diet and exercise, and psychosocial techniques, in addition to medical treatments.

Results:

1) A territorial network coordinated by APMARR was created involving Puglia Region, Health Authorities, Professional board of Psychologists, National
Association of People with FMS, Professional board of Physicians, Italian Society Of Rheumatology-Puglia

2) A communication campaign was widely spread among social network, a brochure was written in collaboration with the Italian Society of Rheumatology and distributed to the general public, a national level conference was organized in Bari

3) A training program acknowledged by the Italian Health Minister Program involved more than $120 \mathrm{GPs}$

4) A patients expert program involved more than 100 patients

5) A group of patients were tutored in their treatment with hyperbaric oxygen therapy and a report was sent to the HTA Italian Program in collaboration with the Puglia Region HTA Center. It was the first HTA report elaborated in collaboration with a patient organization in Italy

6) Self-mutual help groups were organized with free psychological support, as well as an information desk and a toll-free number

7) High satisfaction and pain relief of the participants as detected by a qualitative satisfaction questionnaire
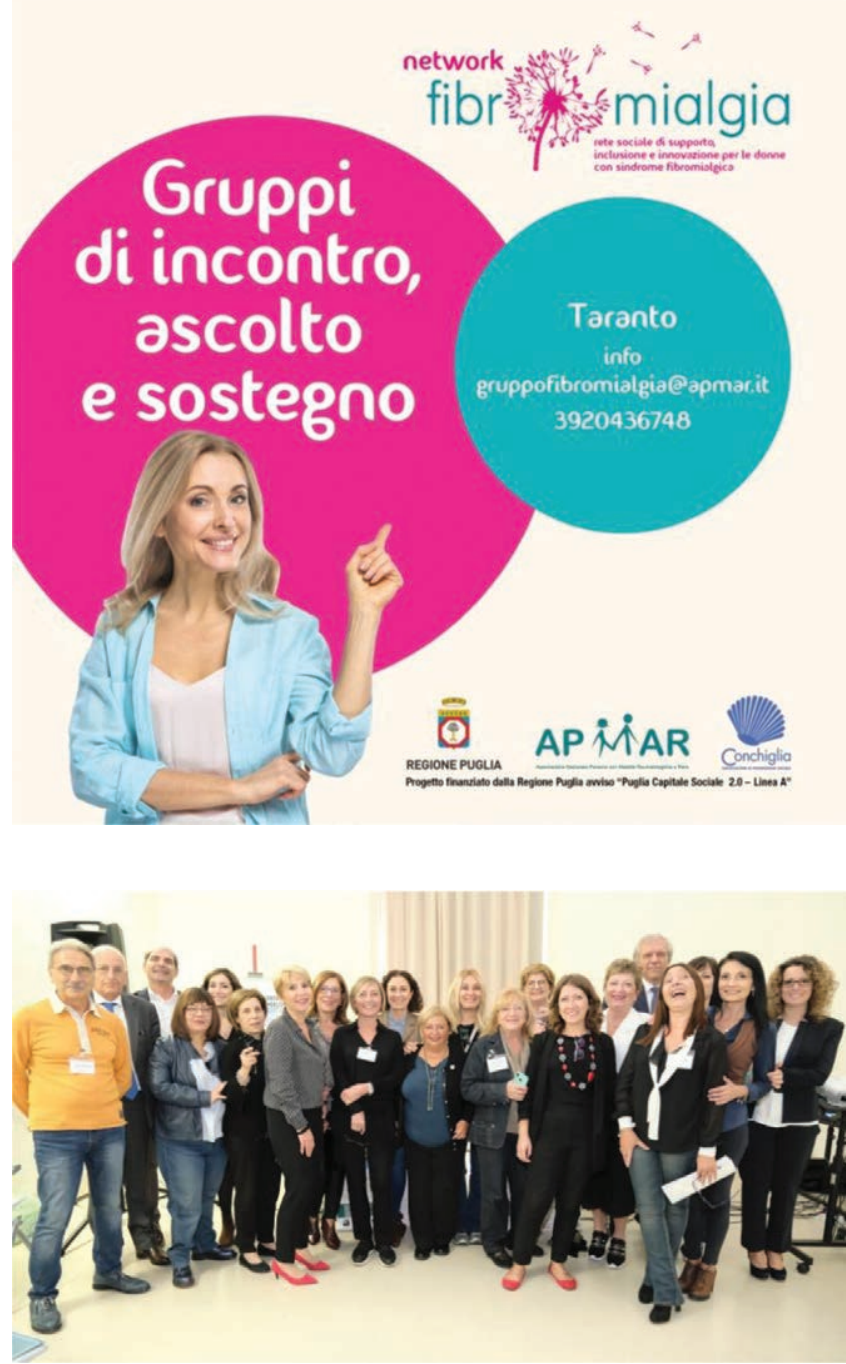

Conclusion: The project demonstrated the good results of the holis tic approach in the patients who took part in the program that reported the improvements of their quality of lives and relieve from their daily pains. The 
Self-mutual help group was the most appreciated free service, in which participants shared personal stories and perspectives thoughtfully and courageously. The training initiatives organized in collaboration with physicians helped them to learn tips for a better lifestyle management, diet and exercise, and psychosocial techniques but above all helped to overcame concerns and frustration regarding the lack of understanding in the medical community. The network succeeds to increased awareness and understanding of FMS across the public opinion and GPs.

References:

[1] Author: S.Mingolla, APMARR Project Manager; Co-authors: A.Celano, APMARR President; I. Cinieri, Psychologist, A. Marsico, Rheumatologist Disclosure of Interests: None declared

DOI: 10.1136/annrheumdis-2020-eular.1782

\section{OP0321-HPR HIGHER QUALITY OF CARE AND LESS SURGERY AFTER IMPLEMENTING OSTEOARTHRITIS GUIDELINES IN PRIMARY CARE- LONG-TERM RESULTS FROM A CLUSTER RANDOMIZED CONTROLLED TRIAL}

N. Osteras ${ }^{1}$, T. Moseng ${ }^{1}$, L. Van Bodegom-Vos ${ }^{2}$, K. Dziedzic ${ }^{3}$, Ø. Andreassen ${ }^{1}$, A. M. Fenstad ${ }^{4}$, O. Furnes ${ }^{4}$, J. Nygren Hansen ${ }^{1}$, B. Natvig ${ }^{5}$, J. H. Røtterud ${ }^{6}$, U. B. Schjervheim ${ }^{7}$, T. P. M. Vliet Vlieland ${ }^{2}$, K. B. Hagen ${ }^{1}{ }^{1}$ Diakonhjemmet Hospital, Oslo, Norway; ${ }^{2}$ Leiden University, Leiden, Netherlands; ${ }^{3}$ Keele University, Keele, United Kingdom; ${ }^{4}$ Haukeland University Hospital, Bergen, Norway; ${ }^{5}$ University of Oslo, Oslo, Norway; ${ }^{6}$ Akershus University Hospital, Lørenskog, Norway; ${ }^{7}$ Nes Municipality, Nes, Norway

Background: To improve quality of care for patients with hip and knee osteoarthritis (OA), a structured model for integrated OA care was developed and implemented among general practitioners (GPs) and physiotherapists (PTs) in primary care. The model was developed based on international treatment recommendations. After 6 months, patient-reported quality of care and satisfaction with care were greater, more patients were referred to physiotherapy and fewer to orthopaedic surgeon, and more patients fulfilled physical activity criteria among OA patients receiving the new model of care compared to the usual care control group ${ }^{1}$.

Objectives: To assess the long-term effects 12 months after implementing the model in primary care.

Methods: A cluster-randomised controlled trial with a stepped-wedge design was conducted in six Norwegian municipalities (clusters). The intervention included implementation of the model, facilitated by interactive workshops for GPs and PTs. The main components of the model were a PT led, 3 hour patient education programme followed by 8-12 weeks of individually tailored, supervised exercise. Patient participants were $\geq 45$ years with symptomatic hip or knee OA. Primary outcome was patient-reported quality of care (OsteoArthritis Quality Indicator questionnaire; $0-100,100=$ optimal quality). Secondary outcomes included satisfaction with care, referrals to physiotherapy, orthopaedic surgeon and magnetic resonance imaging (MRI), joint replacement surgery, fulfilment of physical activity recommendations, and proportion with overweight (body mass index $\geq 25 \mathrm{~kg} / \mathrm{m} 2$ ). Data was analysed using multilevel mixed models adjusted for age, sex and secular time.

Results: In all, 40 of 80 GPs and 37 of 64 PTs attended the workshops. A total of 393 patients with hip and knee OA were included, with 284 in the intervention and 109 in the usual care control group. In the intervention group, 92\% attended the OA education programme and $64 \%$ completed $\geq 8$ weeks of exercise. At 12 months the intervention group reported significantly higher quality of care (score 58 vs. 41 , mean difference: $17.6 ; 95 \% \mathrm{Cl} 11.1,24.0$ ) compared to the control group. The intervention group reported significantly higher satisfaction with care (Odds ratio (OR) $7.8 ; 95 \% \mathrm{Cl} 3.55,17.27$ ) and a significantly larger proportion (OR: $4.0 ; 95 \% \mathrm{Cl} 1.27,12.63$ ) met the recommendations for physical activity compared to the control group. A smaller proportion was referred to orthopaedic surgeon (OR $0.5 ; 95 \% \mathrm{Cl} 0.29,1.00)$ and a smaller proportion received joint replacement surgery in the intervention (4\%) compared to the control group (11\%) (OR $0.3 ; 95 \% \mathrm{Cl} 0.14,0.74)$. The proportion of patients referred to physiotherapy or MRI and the proportion with overweight were similar between the groups.

Conclusion: Implementation of a structured model for OA care led to improved quality of care, higher satisfaction with care and higher physical activity levels after 12 months. These results are comparable to the 6 months results, which indicate a long-term persistence in the beneficial effects of the intervention. The lower surgical rate in the intervention compared to the control group suggests that higher uptake of OA recommendations in primary care may reduce or postpone the need for surgery in people with hip or knee OA.

\section{References:}

[1] Østerås N, Moseng T, Bodegom-Vos LV, et al. Implementing a structured model for osteoarthritis care in primary healthcare: A stepped-wedge cluster-randomised trial. PLOS Medicine. 2019;16(10):e1002949.

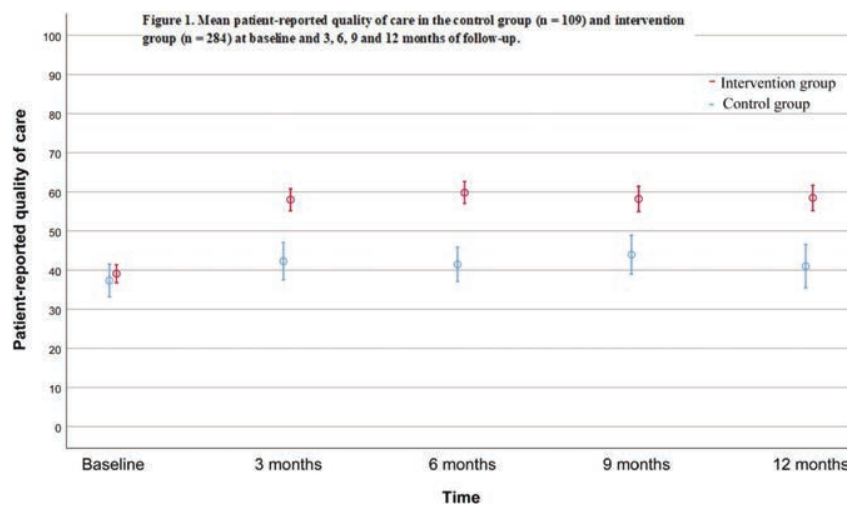

Disclosure of Interests: None declared

DOI: 10.1136/annrheumdis-2020-eular.3575

\section{How to communicate effectively with the patients}

\section{OP0322-PARE HOW TO COMMUNICATE DIAGNOSTIC INFORMATION AND CUTTING EDGE SCIENCE TO PATIENTS WITH RHEUMATIC DISEASES}

I. Loell ${ }^{1}$, S. Jansson ${ }^{1}$, J. Hammarström ${ }^{1}$, L. Alemo Munters ${ }^{1,2} .{ }^{1}$ Swedish Rheumatism Association, Stockholm, Sweden; ${ }^{2}$ Karolinska University Hospital, Functional Area Occupational Therapy and Physical Therapy, Stockholm, Sweden

Background: According to the 2017 Swedish Rheumatology Association (SRA) member strategy a recurring member survey as well as a member withdrawal survey was stipulated. The strategy was developed to evaluate to what extent SRA fulfills the requirements and expectations of its members. According to the 2019 survey, the most important output from a SRA membership, the members rank information about their diagnosis (\#1) and supporting the research of these diagnoses (\#2) most valuable.

Objectives: To transfer the medical and scientific expertise of the rheumatic diagnoses into lay information in order to meet the member's needs; to take part of the results of the cutting edge science and research progress, funded by SRA, that are relevant and important to individuals living with rheumatic conditions.

Methods: A targeted scientific communication strategy was made consisting of lectures, interviews and scientific writing created for multi-channel distribution.

Results: Actions taken upon the survey result

- Brief summaries of every research project funded by SRA in 2019 was written and distributed via social media.

- A research day for lay people was arranged in collaboration with a regional SRA branch and invited speakers. The filmed lectures are also available online.

- A research report with in-depth interviews with researchers and brief summaries about the research funded by SRA was produced. The report was printed and distributed in 70,000 copies to the SRA members, donors and at SRA meetings and conferences.

- Diagnosis sheets aimed to newly diagnosed patients with the most essential information has been developed in collaboration with a patient research partner and an expert researcher within the field. The sheet is printable and can be distributed by any healthcare practitioner or by patients/public.

- Online patient school prototype - gout. In collaboration with the SRA funded gout network we are gathering high quality information about the diagnosis, treatment, self-care and support in the meeting with the healthcare provider for patients to easily navigate and to find robust answers to their inquiries about their disease.

Conclusion: As a member of SRA, regardless of rheumatic disease, the main interest is knowing more about their diagnosis and about the ongoing research in the field. Through collaborations and communicating rheumatic conditions and 